\title{
Mechanisms of Fatty Acid-Induced Insulin Resistance in Muscle and Liver Rafik Ragheb ${ }^{1,2,3,4,5 *}$ and Amina M. Medhat ${ }^{1}$
}

${ }^{1}$ University of Ain Shams - Department of Biochemistry - Faculty of Science, Cairo - Egypt

2University Health Network, Canada

${ }^{3}$ Hospital for Sick Children, Canada

${ }^{4}$ Mount Sinai Hospital, Canada

${ }^{5}$ University of Toronto, Toronto, Canada

\begin{abstract}
Insulin Resistance occurs as a result of disturbances in lipid metabolism and increased levels of circulating fatty acids that accumulate within the insulin sensitive tissues such as muscle, liver and adipose tissues. Increased fatty acid flux has been suggested to be strongly associated with insulin resistant states such as obesity and type 2-diabetes. Fatty acids appear to cause this defect in glucose transport by inhibiting insulin -stimulated tyrosine phosphorylation of insulin receptor substrate-1 (IRS-1) and reducing IRS-1 associated phosphatidyl-inositol 3-kinase activity that implicate other insulin signaling components downstream of the insulin signaling cascade. A number of different metabolic abnormalities may increase intramyocellular or intrahepatic fatty acid metabolites that induce the disease state of insulin resistance through a number of different cellular mechanisms. The current review point out the link between enhanced FFA flux and activation of PKC and how it impacts on both the insulin signaling in muscle and liver.
\end{abstract}

Keywords: Clinical biochemistry; Molecular endocrinology; Insulin resistance; Free Fatty Acids (FFAs) and type 2 diabetes

\section{Introduction}

Diabetes is a chronic metabolic disorder affecting $\sim 5 \%$ of the population in the industrialized nations as it is becoming a huge concern worldwide. In Canada about $\sim 1.3$ million ( $4.9-5.8 \%$ of the total Canadian population) aged greater than or equal to 12 years have diabetes [1]. In another part of the world, it is estimated that Diabetes Mellitus is a major emerging clinical and public problem in a country such as Egypt among others [2].

Lack of or severe reduction in insulin secretion due to autoimmune destruction of $\beta$-cell is responsible for type I diabetes mellitus. However, the more prevalent form is type 2 diabetes; it represents more than $90 \%$ of the cases [3]. The pathogenesis of type 2 diabetes is complex, involving progressive development of insulin resistance and a relative deficiency in insulin secretion that is the leading cause for the onset of hyperglycemia. This review outlines and focuses on the specific role of FFA that may contribute to the development of muscle and hepatic insulin resistance. Further knowledge and exploration of the molecular mechanisms involved are of substantial interest for future therapeutic interventions as well as in determining the high risk individuals through the discovery of novel diagnostic biomarkers.

Type 2 diabetes mellitus is characterized by increased hepatic glucose production (HGP), the inability of insulin to increase the uptake of glucose (peripheral insulin resistance) and suppress HGP (hepatic insulin resistance), and impairment of insulin secretion [4-7].

Obesity is associated with insulin resistance as one of the key features of type 2 diabetes, mainly due to the release of free fatty acids (FFA) [8-12] and secondary the release of inflammatory cytokines [13] from the expanded adipose tissue mass (8-13). Studies conducted from our own and others (i.e., Drs. Defronzo and Reaven) laboratories have clearly shown that individuals with obesity [14-19], type 2 diabetes (20) and obesity-associated type 2 diabetes $[15,17,20]$ have elevated plasma FFA levels.

Not only are the plasma FFA levels elevated in obesity; there is also an increased FFA flux. The increased FFA flux is mainly due to increased lipolysis from the expanded adipose tissue stores, to resist the insulin's antilipolytic action and increase sensitivity to lipolytic hormones [21] Numerous studies have shown that FFAs are an important causative link between obesity, insulin resistance and type 2 diabetes mellitus [ 4 8,10,11-13]. Elevation of plasma FFA has been shown to impair insulin action, and to be a risk factor for the development of type 2- diabetes [22].

\section{Insulin resistance}

Insulin resistance is well defined as the decreased ability of insulin to regulate glucose metabolism. It is an important target of medical research as it represents a common disorder/disease in a range of metabolic diseases that are termed as Metabolic Syndrome. It includes type 2 diabetes, glucose intolerance, dyslipidaemia, hypertension and cardiovascular diseases. Insulin action starts when insulin binds to its receptor at the cell surface to initiateits effect through signaling molecules. Upon insulin binding to its receptor, insulin receptor tyrosine kinase activity is activated, which results in receptor autophosphorylation. The activated insulin receptor tyrosine kinase also phosphorylates insulin receptor substrates, which include insulin receptor substrate (IRS) 1- 4 and Shc (one of the MAPK pathway components). Phosphorylated IRS 1-4 mainly activate the phosphatidylinositol 3-kinase (PI3K) pathway, and Shc mainly activates the Grb2/Sos ( a downstream proteins in the MAPK pathway). The skeletal muscle is responsible for about $80 \%$ of whole body insulin stimulated glucose uptake [23]. The specific cause of reduced insulin action in skeletal muscle cells is unclear. It is aberrant that the disregulated lipid metabolism and the increased free fatty acids have a strong implication.

*Corresponding author: Rafik Ragheb, University of Toronto, Toronto - Canada, Tel/Fax: 1-905-2099660; E-mail: rafik.ragheb@utoronto.ca

Received April 06, 2011; Accepted May 13, 2011; Published May 16, 2011

Citation: Ragheb R, Medhat AM (2011) Mechanisms of Fatty Acid-Induced Insulin Resistance in Muscle and Liver. J Diabetes Metab 2:127. doi:10.4172/21556156.1000127

Copyright: @ 2011 Ragheb R, et al. This is an open-access article distributed unde the terms of the Creative Commons Attribution License, which permits unrestricted use, distribution, and reproduction in any medium, provided the original author and source are credited. 
The link between increased lipid availability and insulin resistance: Randle et al. (1963) was the first to suggest a primary role for elevated FFA availability and the development of insulin resistance. Randel speculated that high plasma concentration of FFA is one of the common characteristics in patients with either diabetes or other carbohydrate disorders [24,25]. Randle's hypothesis was supported by different studies, it manifested that insulin resistance can be induced within hours through lipid infusion or weeks through a high fat feeding regimen. In addition, studies of a number of genetic experimental models of insulin resistance also implicate the role of increased lipid availability in the pathogenesis of the disease.

The glucose fatty acid cycle: The scientific basis of Randle's hypothesis is based on the increased FFA oxidation. As a result, the mitochondrial acetyl-CoA/CoA ratio increases that causes a reduction in the supply of acetyl -CoA from pyruvate. This leads to the increase of citrate concentration that would cause an accumulation of glucose 6-phosphate that result in inhibition of hexokinase and the uptake of glucose by the cell $[24,25]$.

Although lipid infusion results in an inhibition of glucose oxidation, the insulin stimulated glucose uptake appears with no effect for hours. Apparently, the impairment effect is implicated on the glycogen synthesis instead. In addition, the reduction in glycogen synthesis occurs with a decreased level of glucose 6-phosphate rather than the accumulation and increase in its level. The current consensus has been attributed to the reduction of glucose transport and phosphorylation in association with a reduction in the activity of the insulin signaling cascade [26,27]. These are the main reasons that shed a light for the involvement of other mechanisms in the development of insulin resistance.

Early studies have pointed out that high fat feeding result in an accumulation of intracellular triglyceride as a major factor involved in insulin resistance. This is now looked as one of most consistent markers of whole body insulin resistance. Other major contributors for causing the onset of insulin resistance are the lipid intermediates such as long -chain fatty acyl CoAs (LCACoAs), diacylglycerol (DAG) and ceramides.

LCACoAs: are to represent the activated form of intracellular FFA [28]. LCACoAs are seen as signaling molecules that affect a variety of cellular processes for example, it inhibit the hexokinase activity in muscle in vitro. LCACoAs were seen to interfere with the muscle glucose utilization through the activation of Protein Kinase C (PKC) [29]. In addition, LCACoAs can modulate gene transcription such as the hepatic nuclear factor $4 a[30]$.

DAG: is an intermediate of both triglyceride and phospholipids metabolism that accumulate in the muscle insulin resistant rat model following high fat feeding [31]. DAG can be generated by de novo synthesis following the estrification of LCACoAs to glycerol3-phosphate or through the break down of phospholipids such as phosphatidylinositol-4,5-bisphosphate and phospatidylcholine by phospholipases C and D. DAG act as important second messenger involved in intracellular signaling in addition to its effect on insulin action by activating PKC [32].

Ceramides: is a derivative of sphingomyelin, the phosphorlipid component for cell membranes that is synthesized through the action of sphingomyelinase from the palmitoyl CoA. Ceramides act as a second messenger that can alter the activity of kinases, phosphatases and transcription factors that regulate a number of processes such as proliferation, differentiation and apoptosis.
Our own data and others have demonstrated that palmitate induce insulin resistance in muscle model through the reduction of IRS-1 and $\mathrm{PKB}$ phosphorylation and activity as a result of activation of protein phosphatase 2A (PP2A) [33]. Other reports have shown that ceramides can mediate the inhibition of insulin signaling through the tumor necrosis factor $\alpha(\mathrm{TNF} \alpha)$, an activator of sphingomylinase [34].

Data from our laboratory have clearly demonstrated the effect of FFA induced insulin resistance in two insulin sensitive tissues muscle and liver. The key findings are summarized as follow:

1- Free fatty acids (FFAs) - induced PKC and NFKB activation, two key events in two different models for insulin resistance, the skeletal muscle and liver [35,37].

2- In the $\mathrm{C} 2 \mathrm{C} 12$ muscle insulin resistant model, different Free fatty acids (FFAs) - induced serine 307 phosphorylation for IRS-1 as a mechanism for skeletal muscle insulin resistance [35]. The inhibitory effects of PKC on insulin signaling may at least in part be explained by the serine/threonine phosphorylation of IRS1. Both oleate and palmitate treatment were able to increase the serine 307 phosphorylation of IRS-1 (Figure 1). IRS-1 serine 307 phosphorylation is inducible which causes the inhibition of IRS1 tyrosine phosphorylation by either IkB-kinase (IKK) or c-jun N-terminal kinase (JNK) [35,36].

3- In the fructose fed hamster model of hepatic insulin resistance, free fatty acids - induced PKC and NFKB activation as a mechanism for both insulin resistance and dyslipidemia [37] (Figure 2).

Our in vitro and in vivo key findings generally support the relevance and the importance to the human insulin resistance scenario. Strong body of evidences and our own data have pointed out the involvement of the IKK-beta/IKappa B/NFKappa B pathway in insulin resistance in the two models of muscle and liver [35,37].

Furthermore, we are among the first laboratory that manifested a direct evidence for FFA induced insulin resistance through the seine phosphorylation of IRS-1 in $\mathrm{C} 2 \mathrm{C} 12$ skeletal muscle cells that compromised the IRS-1 phosphorylation as seen in our data. The finding requires further attention as it represents a therapeutic target for the disease intervention.

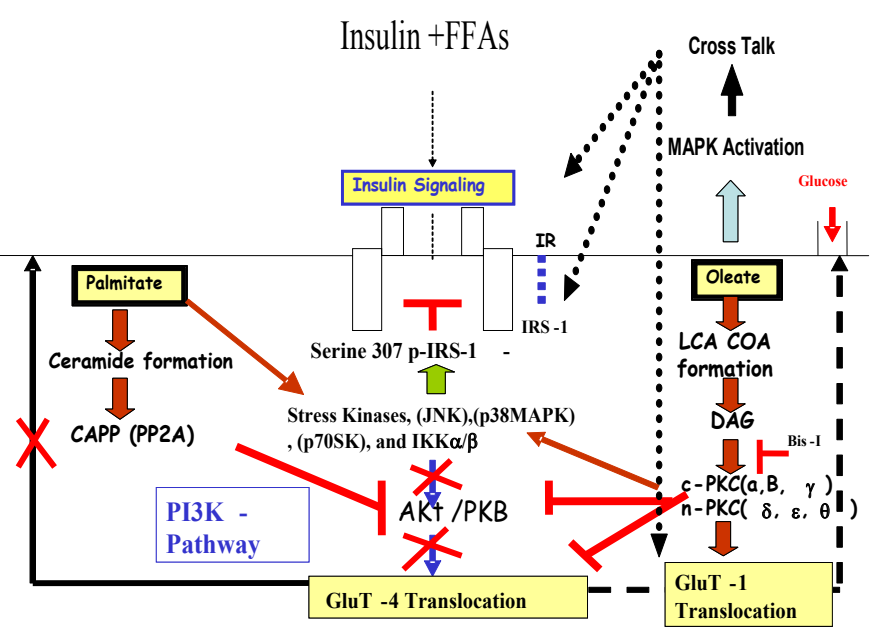

Figure 1: Hypothetical scheme to explain changes in the insulin signaling upon treating the $\mathrm{C} 2 \mathrm{C} 12$ skeletal muscle cells with different species of FFAs, oleate and palmitate [35]. 


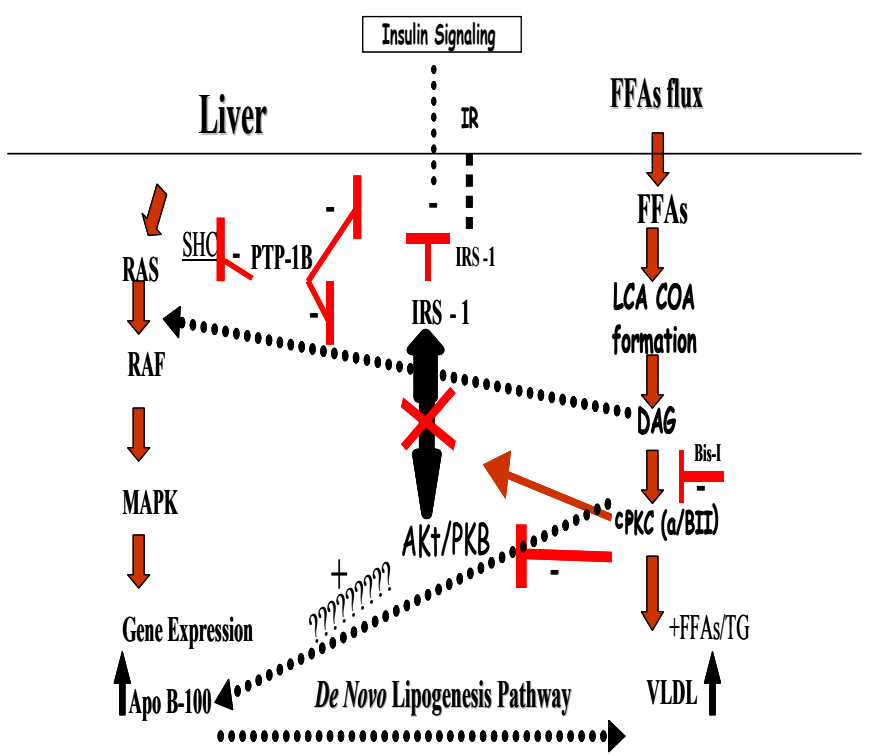

Figure 2: Hypothetical scheme explaining the changes in insulin signaling and de novo lipogenesis following two weeks of fructose feeding as a model for hepatic insulin resistance [36-37].

On the other hand, we also have reported that increased fatty acid flux to the liver that resulted PKC activation in the fructose-fed model are important events contributed to hepatic apoB100 overproduction commonly observed in insulin resistant states [37]. These current data point to FFA-induced PKC activation and its impact on dyslipidemia as a potential therapeutic target in the treatment of diabetes and insulin resistance.

\section{Summary}

Normal cellular fatty acid homeostasis reflects a balance between processes that generate or deliver fatty acids and processes that utilize these molecules. In mammalian cells, free fatty acids are generated through the de-novo synthetic pathway and liberated when triglycerides and phospholipids are hydrolyzed by cellular lipases [38]. High plasma FFA and triglyceride levels lead to increased import of FFA into nonadipose tissues, contributing to intracellular lipid accumulation. Non-adipose tissues such as liver and skeletal muscle [39] have a limited capacity for lipid storage leading to cellular dysfunction, the phenomenon that is termed lipotoxicity. Studies have documented that primary hyperlipidemia, serum triglycerides $[40,41]$ and FFA $[42,43]$ are elevated in type 1 and type 2 diabetes and plasma FFA are elevated in obese individuals [44]. Insulin resistance is believed to be the primary cause of type 2 diabetes. Recent studies show that elevated plasma levels of free fatty acids (FFA) might increase insulin resistance in muscle and liver. Lowering of FFA levels is therefore postulated to be a potential therapeutic target for type 2 diabetes.

The aim of current review article and the studies conducted in our laboratory was to further elucidate the hypothesis that "Increased lipid availability induces perturbations in key molecules of the insulinsignaling pathway leading to a reduced insulin action in insulin sensitive tissues such as muscle and liver. Free fatty acid induced insulin resistance may be an important underlying factor in the development of insulin resistant states such as type 2 diabetes". We focused on elucidating the molecular mechanisms by which FFA induce insulin resistance in two insulin sensitive tissues muscle and liver. Specifically, we investigated in details the mechanisms and the effects of two FFAs, the oleate, the monounsaturated FFA (18:1) and palmitate, the saturated FFA (16:00) as they are the most predominant two fatty acids in the circulation (45). We studied their effect on glucose metabolism and insulin action in two insulin sensitive tissues the muscle and the liver. We investigated how FFAs can induce the insulin resistance at the molecular level in vitro and in vivo in two different experimental designs for insulin resistance.

Several mechanisms that affect insulin signal transduction have been identified. In our laboratory we found that oleate does not affect the total protein level of $\mathrm{PKB} / \mathrm{Akt}$ total but partially reduces the phosphorylation of $\mathrm{PKB}$ in oleate and dramatically reduced in palmitate treated cells. On the other hand, our data suggest that the monounsaturated fatty acid; oleate muscle insulin resistance is mainly via PKC. In the contrary, the saturated fatty acid, palmitate induces insulin resistance mechanistically through the PKB inhibition that is well documented. In addition, the two FFAs are also to activate the NFKB and the stress kinases that contributed to the induction of serine 307 phosphorylation of IRS-1 and the development of insulin resistance in the $\mathrm{C} 2 \mathrm{C} 12$ muscle model. Recently, Wang et al. have reported that palmitate specifically induced insulin resistance by PKC theta-dependent activation of mTOR/S6K pathway in C2C12 myotubes [46] However, more studies are still required to further elucidate the exact consequences of PKC activation following other species of FFA (s) treatment [47].

Furthermore, our second set of data is to represent the experimental work that was conducted in the fructose fed hamster as a liver model for diet induced insulin resistance. The data points FFA - induced PKC activation and its impact on dyslipidemia seen in the liver model. This model of liver insulin resistance has revealed the importance of PKC activation for both insulin resistance and de novo lipogenesis.

Ragheb el al. have demonstrated that the antioxidant, Taurine was capable of reversing the oleate-induced insulin resistance in myocytes as manifested from the glucose uptake data [35]. Han et al. have also reported that Taurine prevented free fatty acids-induced hepatic insulin resistance in association with inhibiting JNK1 activation and improving insulin signalling in vivo [48]. As a result, Taurine is considered as a potential therapeutic target in protecting from insulin resistance caused by elevated FFAs through different mechanisms of oxidative stress and inflammatory pathways. Gao et al. have reported that oxidative stress acts as a link between FFA and hepatic insulin resistance. NADPH oxidase 3 (NOX3) - derived reactive oxygen species (ROS) may mediate the effect of TNF-alpha on hepatocytes in $\mathrm{db} / \mathrm{db}$ mice and Hep G2 cells treated with palmitate. It was found that palmiate induced hepatic insulin resistance through JNK and p38MAPK pathways that are rescued by siRNA-mediated NOX3 reduction [49]. And, Klip et al. have demonstrated that proinflammatory activation within adipose tissue of obese and high fat fed animals and humans causes muscle insulin resistance and is ascribed to circulating inflammatory cytokines. Recent evidence also shows proinflammatory macrophages infiltrating muscle tissue and or intermuscular adipose tissue and there is growing evidence that FFA trigger macrophages to secrete factors that directly impair insulin actions [50].

\section{Conclusion}

This short review has outlined aspects of FFA - induced insulin resistance in insulin sensitive tissues. It is clear that the development of insulin resistance and metabolic disease is marked by the disturbances of lipids in the body. Furthermore, the data have demonstrated the importance of PKC activation and inflammation in FFA induced 
insulin resistance. In 1997, the WHO has recognized the importance of insulin resistance and its link to type 2 diabetes, this growing problem that has a great deal for the public health globally. Further insight to the field will be invaluable and of great impact on the treatment and the early intervention and prevention of the disease onset. In addition, controlling FFAs levels in plasma would benefit patients with type 2 - diabetes and obesity. Further, this approach of therapy and early prevention has a potential role in controlling the ongoing epidemic of type 2- diabetes worldwide.

\section{Acknowledgements}

Authors are grateful to Professor Dr. Fantus I.G. and Dr. K. Adeli for their great efforts and guidance as consultant experts in the field of insulin resistance and metabolic dyslipidemia with our research work (Toronto, Canada). In addition, we acknowledge the great efforts of Professors Dr. Gamila M. Shanab and Dr. Dina M. Seoudi from Ain Shams University (Cairo, Egypt).

\section{Conflict of Interest}

Rafik Ragheb is a recipient of the Hospital for Sick Children Research Training Competition (Restra-comp) - The University of Toronto, Canada.

\section{References}

1. Dawson KG, Gomes D, Gerstein H, Blanchard JF, Kahler KH (2002) The economic cost of diabetes in Canada, 1998. Diabetes Care 25: 1303-1307.

2. Galal OM (2002) The nutrition transition in Egypt: obesity, undernutrition and the food consumption context. Public Health Nutr 5: 141-148.

3. Harris MI, HaddenWC, KnowlerWC, Bennett PH (1987) Prevalence of diabetes and impaired glucose tolerance and plasma glucose levels in U.S. population aged 20-74 yr. Diabetes 36: 523-534.

4. Defronzo RA (1988) Lilly lecture 1987. The triumvirate: beta-cell, muscle, liver. A collusion responsible for NIDDM. Diabetes 37: 667-687.

5. Defronzo RA. (1992) Pathogenesis of type 2 (non-insulin dependent) diabetes mellitus: a balanced overview. Diabetologia 35: 389-397.

6. Defronzo RA, Bonadonna RC, Ferrannini E (1992) Pathogenesis of NIDDM. A balanced overview. Diabetes Care 15: 318-368.

7. Kahn CR (1994) Banting Lecture. Insulin action, diabetogenes, and the cause of type II diabetes. Diabetes 43: 1066-1084.

8. Boden G (1997) Role of fatty acids in the pathogenesis of insulin resistance and NIDDM. Diabetes 46: 3-10.

9. Henry RR (1995) Impaired muscle fat metabolism: a cause or effect of visceral obesity? J Clin Invest 95: 1427-1428.

10. Kelley DE, Mandarino LJ (2000) Fuel selection in human skeletal muscle in insulin resistance: a reexamination. Diabetes 49: 677-683.

11. Lewis GF, Carpentier A, Adeli K, Giacca A (2002) Disordered fat storage and mobilization in the pathogenesis of insulin resistance and type 2 diabetes. Endocr Rev 23: 201-229.

12. McGarry JD (1998) Glucose-fatty acid interactions in health and disease. Am J Clin Nutr 67: 500S-504S

13. Kern PA, Ranganathan S, Li C, Wood L, Ranganathan G (2001) Adipose tissue tumor necrosis factor and interleukin-6 expression in human obesity and insulin resistance. Am J Physiol Endocrinol Metab 280: E745-E751.

14. Bolinder J, Kerckhoffs DA, Moberg E, Hagstrom-Toft E, Arner P (2000) Rates of skeletal muscle and adipose tissue glycerol release in nonobese and obese subjects. Diabetes 49: 797-802.

15. Carpentier A, Mittelman SD, Bergman RN, Giacca A, Lewis GF (2000) Prolonged elevation of plasma free fatty acids impairs pancreatic betacell function in obese nondiabetic humans but not in individuals with type 2 diabetes. Diabetes 49: 399-408.

16. Coppack SW, Evans RD, Fisher RM, Frayn KN, Gibbson GF, et al. (1992) Adipose tissue metabolism in obesity: lipase action in vivo before and after a mixed meal. Metabolism 41: 264-272.

17. Felber JP, Ferrannini E, Golay A, Meyer HU, Theiband D, et al. (1987) Role of lipid oxidation in pathogenesis of insulin resistance of obesity and type I diabetes. Diabetes 36: 1341-1350.

18. Laws A, Hoen HM, Selby JV, Saad MF, Haffner SM, et al. (1997) Differences in insulin suppression of free fatty acid levels by gender and glucose tolerance status. Relation to plasma triglyceride and apolipoprotein B concentrations. Insulin Resistance Atherosclerosis Study (IRAS) Investigators. Arterioscler Thromb Vasc Biol 17: 64-71.

19. Reaven GM, Hollenbeck C, Jeng CY, Wu MS, Chen YD (1988) Measurement of plasma glucose, free fatty acid, lactate, and insulin for $24 \mathrm{~h}$ in patients with NIDDM. Diabetes 37: 1020-1024.

20. Baldeweg SE, Golay A, Natali A, Balkau B (2000) Insulin resistance, lipid and fatty acid concentrations in 867 healthy Europeans. European Group for the Study of Insulin Resistance (EGIR). Eur J Clin Invest 30: 45-52.

21. Bjorntorp P, Bergman H, Varnauskas $E$, Lindholm B (1969) Lipid mobilization in relation to body composition in man. Metabolism 18: 840-851.

22. Paolisso G, Tataranni PA, Foley JE, Bogardus C, Howard BV, et al. (1995) A high concentration of fasting plasma non-esterified fatty acids is a risk factor for the development of NIDDM. Diabetologia 38: 1213-1217.

23. DeFronzo RA, Jacot E, Jequier E, Maeder E, Wahren J, et al. (1981)The effect of insulin on the disposal of intravenous glucose. Results from indirect calorimetry and hepatic and femoral venous catheterization. Diabetes 30 1000-1007.

24. Randle P, Garland PB, Hales CN, Newsholme E (1963) The glucose fatty-acid cycle. Its role in insulin sensitivity and the metabolic disturbances of diabetes mellitus. Lancet 13: 785-789.

25. Randle PJ, Newsholme EA, Garland PB (1964) Regulation of glucose uptake by muscle. 8 . Effects of fatty acids, ketone bodies and pyruvate, and of alloxandiabetes and starvation, on the uptake and metabolic fate of glucose in rat heart and diaphragm muscles. Biochem J 93: 652-665.

26. Boden G, Chen X, Ruiz J, White JV, Rossetti L (1994) Mechanisms of fatty acid-induced inhibition of glucose uptake. J Clin Invest 93: 2438-2446.

27. Roden M, Price TB, Perseghin G, Petersen KF, Rothman DL, et al. (1996) Mechanism of Free Fatty Acid-induced Insulin Resistance in Humans. J Clin Invest 97: 2859-2865.

28. Chen MT, Kaufman LN, Spennetta T, Shrago E (1992) Effects of high fatfeeding to rats on the interrelationship of body weight, plasma insulin, and fatty acyl-coenzyme A esters in liver and skeletal muscle. Metabolism 41: 564-569.

29. Nishizuka $Y$ (1995) Protein kinase $C$ and lipid signaling for sustained cellular responses. FASEB J 9: 484-496.

30. Hertz R, Magenheim J, Berman I, Bar-Tana J (1998) Fatty acyl-CoA thioesters are ligands of hepatic nuclear factor-4alpha. Nature 392: 512-516.

31. Oakes ND, Kennedy CJ, Jenkins AB, Laybutt DR, Chisholm DJ, et al. (1994) A new antidiabetic agent, BRL 49653, reduces lipid availability and improves insulin action and glucoregulation in the rat. Diabetes 43: 1203-1210.

32. Schmitz-Peiffer C (2000) Signalling aspects of insulin resistance in skeleta muscle: mechanisms induced by lipid oversupply. Cell Signal 12: 583-594

33. Schmitz-Peiffer C, Craig DL, Biden TJ (1999) Ceramide generation is sufficient to account for the inhibition of the insulin-stimulated PKB pathway in $\mathrm{C} 2 \mathrm{C} 12$ skeletal muscle cells pretreated with palmitate. J Biol Chem 274: 24202-24210.

34. Cazzolli R, Carpenter L, Biden TJ, Schmitz-Peiffer C (2001) A role for protein phosphatase $2 A$-like activity, but not atypical protein kinase Czeta, in the inhibition of protein kinase B/Akt and glycogen synthesis by palmitate. Diabetes 50: 2210-2218.

35. Ragheb R, Shanab GML, Medhat MA, Seoudi DM, Adeli K, et al. (2009) Free fatty Acids - induced muscle insulin resistance and glucose uptake dysfunction: Evidence for PKC activation and oxidative stress activated signaling pathways. Biochem Biophys Res Commun 389: 211-216.

36. Ragheb R, Medhat AM, Shanab GML, Seoudi DM, Fantus IG (2009) Prolonged Treatment with free fatty acids has post receptor effect in hepatic insulin resistance: Evidence that free fatty acids, oleate and palmitate have insignificant effect on the insulin receptor beta in vivo and ex vivo primary hepatocytes. Biochem Insights 2: 29-36.

37. Ragheb R, Medhat AM, Shanab GML, Seoudi MS, Fantus IG (2008) Links between enhanced Fatty Acid Flux, Protein Kinase C and NFB Activation, and ApoB-Lipoprotein Production in the Fructose Fed Hamster Model of Insulin Resistance. Biochem Biophys Res Commun 370: 134-139. 
Citation: Ragheb R, Medhat AM (2011) Mechanisms of Fatty Acid-Induced Insulin Resistance in Muscle and Liver. J Diabetes Metab 2:127. doi:10.4172/2155-6156.1000127

38. Schaffer JE (2002) Fatty acid transport: the roads taken. Am J Physiol Endocrinol Metab 282: E239-E246.

39. Garg A (2000) Lipodystrophies. Am J Med 108: 143-152.

40. Hallgren B, Stenhagen S, Svanborg A, Svennerholm L (1960) Gas chromatographic analysis of the fatty acid composition of the plasma lipids in normal and diabetic subjects. J Clin Invest 39: 1424-1434.

41. Laakso M, Pyorala K, Voutilainen E, Aro A, Pyörälä K, et al. (1985) Serum lipids and lipoproteins in middle-aged non-insulin-dependent diabetics. Atherosclerosis 56: 271-281.

42. Fraze E, Donner CC, Swislocki ALM, Chiou YA, Chen YD, et al. (1985) Ambient plasma free fatty acid concentrations in noninsulin-dependent diabetes mellitus: evidence for insulin resistance. J Clin Endocrinol Metab 61: 807-811.

43. Zuniga-Guajardo S, Zinman B (1985) The metabolic response to the euglycemic insulin clamp in type I diabetes and normal humans. Metabolism 34: 926-930.

44. Campbell PJ, Carlson MG, Nurjhan N (1994) Fat metabolism in human obesity. Am J Physiol 266: E600-E605.
45. Leskinen MH, Solakivi T, Kunnas T, Alho H, Nikkari ST (2005) Serum fatty acids in postinfarction middle-aged men. Scand J Clin Lab Invest 65: 485-490.

46. Wang X, Yu W, Nawaz A, Guan F, Sun S, et al. (2010) Palmitate induced insulin resistance by $\mathrm{PKC}$ theta-dependent activation of $\mathrm{mTOR} / \mathrm{S} 6 \mathrm{~K}$ pathway in $\mathrm{C} 2 \mathrm{C} 12$ myotubes. Exp Clin Endocrinol Diabetes 118: 657-61.

47. Dasgupta S, Bhattacharya S, Maitra S, Pal D, Majumdar SS, et al. (2011) Mechanism of lipid induced insulin resistance: Activated PKCE is a key regulator. Biochim Biophys Acta 1812: 495-506.

48. Wu N, Lu Y, He B, Zhang Y, Lin J, et al. (2010) Taurine prevents free fatty acidinduced hepatic insulin resistance in association with inhibiting JNK1 activation and improving insulin signaling in vivo. Diabetes Res Clin Pract 90: 288-296.

49. Gao D, Nong S, Huang X, Lu Y, Zhao H, et al. (2010) The effects of palmitate on hepatic insulin resistance are mediated by NADPH Oxidase 3-derived reactive oxygen species through JNK and p38MAPK pathways. J Biol Chem 285: 29965-29973.

50. Kewalramani G, Bilan PJ, Klip A (2010) Muscle insulin resistance: assault by lipids, cytokines and local macrophages. Curr Opin Clin Nutr Metab Care 13: 382-390. 